\title{
Status and challenges of simulations with dynamical fermions
}

\section{Stefan Schaefer*}

CERN, Physics Department, 1211 Geneva 23, Switzerland

E-mail: stefan.schaeferecern.ch

\begin{abstract}
An overview of the current state of algorithms for dynamical fermion simulations is given. In particular some insight into the functioning of the determinant splitting techniques is discussed. The critical slowing down of the simulations towards the continuum limit and the role of the boundary conditions is also reviewed.
\end{abstract}

The 30th International Symposium on Lattice Field Theory

June 24-29, 2012

Cairns, Australia

${ }^{*}$ Speaker. 


\section{Introduction}

Continuous work on algorithms has improved our ability to perform simulations of QCD at light quark masses and on fine lattices. This was possible due to progress in many domains: solvers for the Dirac equation with only a mild scaling towards the chiral limit have been developed as well as better representations of the quark determinant that allow larger step sizes in the integration of the molecular dynamics equations of motion. These methods are combined with reweighting techniques to stabilize the simulations and many other improvements concerning the numerical and theoretical aspects of a simulation. At the same time, the computer hardware available to these computations has become much more powerful too.

Understanding these developments and bringing them together in efficient packages has reduced the cost of current light quark algorithms over the methods available roughly a decade ago by orders of magnitude. A review of some of these methods and an approach towards a theoretical understanding of the determinant splitting techniques is presented in the first part of this contribution.

The focus of these developments has been the ability to simulate light quarks, but for controlled results, also taking the continuum limit is essential. This has so far been hindered by the problem of the freezing topological charge as the lattice spacing is decreased. Practically, this makes large volume simulations below $0.05 \mathrm{fm}$ exceedingly difficult, because below this value, the autocorrelations associated with the topological charge become much longer than typical run lengths and reliable measurements are therefore impossible.

A solution to this problem is to choose a setup where topological sectors do not form in the continuum limit, by using open boundary conditions in time. With these boundary conditions, the topological observables show, in numerical simulations with pure gauge theory, precisely the scaling of the autocorrelation times as expected from the algorithm, i.e., the $\tau_{\text {int }}$ rise with the inverse lattice spacing squared. This then provides a basis for the estimation of the required run length as the simulations move towards the continuum limit, as will be explained in the second part of this writeup.

\section{Update algorithms}

Practically all current simulations with dynamical fermions are performed using a variant of the Hybrid Monte Carlo algorithm (HMC)[1]. Here the gauge field update is achieved by introducing momenta $\pi$ conjugate to the field variables and numerically integrating the molecular dynamics (MD) equations of motion derived from the Hamiltonian

$$
H[\pi, U]=\frac{1}{2}(\pi, \pi)+S_{g}[U]+S_{f}[U],
$$

where $S_{g}[U]$ and $S_{f}[U]$ give the gauge and fermion part of the action.

The numerical integrators for the molecular dynamics equations of motion are not exact, but lead to an energy violation $\delta H=H_{2}-H_{1}$ after a certain molecular dynamics time $\tau$, with $H_{1}$ and $H_{2}$ being, respectively, the value of $H$ at the beginning and the end of the trajectory. Despite this integration error, the algorithm is made exact with a Metropolis step, where the configuration is 
accepted with probability

$$
P_{\text {acc }}=\min \{1, \exp (-\delta H)\} .
$$

It turns out that a higher acceptance rate can not only be reached with a smaller step size and by tuning the integrator of the MD equations of motion, but also by using the flexibility in the representation of the fermion determinant. It is in particular this choice of $S_{f}$, or more precisely the factorization of the fermion matrix, where the various fermion algorithms, like Hasenbusch's mass splitting, the RHMC or the DD-HMC, differ.

As will become clear in the following, the crucial point is that one cannot discuss the merits of different effective fermion actions on their own, without taking into account the interplay between the action and the integrator. We therefore first introduce the integration algorithms in Sec. 2.1 and how to analyze their performance using the shadow Hamiltonian in Sec. 2.2. In this framework, we can then analyze the effect of the determinant splitting (Sec. 2.4), which we demonstrate for the Hasenbusch decomposition in Sec. 2.6.

\subsection{Integration algorithms}

The HMC algorithm requires the use of symplectic and reversible integrators, which are conveniently constructed by alternating updates of the fields $T_{U}$ and the momenta $T_{\pi}$. A popular second order integrator[2] is given by

$$
I=\left\{T_{U}(\delta \tau \lambda) T_{\pi}(\delta \tau / 2) T_{U}(\delta \tau(1-2 \lambda)) T_{\pi}(\delta \tau / 2) T_{U}(\delta \tau \lambda)\right\}^{n}
$$

with

$$
\begin{aligned}
& T_{\pi}:(\pi, U) \rightarrow\left(\pi^{\prime}, U^{\prime}\right)=(\pi-F(U) \delta \tau, U) \\
& T_{U}:(\pi, U) \rightarrow\left(\pi^{\prime}, U^{\prime}\right)=(\pi, U+\pi \delta \tau),
\end{aligned}
$$

with step size $\delta \tau=\tau / n$ and a tunable parameter $\lambda$. Here we only give a single time-scale and a single force $F_{x, \mu}^{a}=\partial_{x, \mu}^{a} S$, but multiple time scale integrators are frequently used[3] and will be briefly discussed in Sec. 2.7.

The many force components and integrator options can lead to a bewildering number of choices. Theoretical understanding that can facilitate the analysis of this optimization problem comes from the theory of symplectic integrators. For a given Hamiltonian, the integrator conserves a so-called shadow Hamiltonian, which can be constructed as a power series in the step-size $\delta \tau$. Even though the radius of convergence of this series is not clear, in practical applications the first order of this series turns out to give a good approximation at least for reasonably small step sizes, as has been shown in a series of papers by Clark et al., whose line of argumentation we follow $[4,5,6,7]$.

For the integrator in Eq. 2.4, the shadow Hamiltonian up to $\delta \tau^{2}$ reads

$$
\widetilde{H}=H+\delta \tau^{2}\left\{c_{1} \sum_{x, \mu} \partial_{x, \mu}^{a} S \partial_{x, \mu}^{a} S-c_{2} \sum_{\substack{x, \mu \\ y, v}} \pi_{x, \mu}^{a} \pi_{y, v}^{b} \partial_{x, \mu}^{a} \partial_{y, v}^{b} S\right\}+\cdots \equiv H+\Delta H
$$

with coefficients $c_{1}=\left(6 \lambda^{2}-6 \lambda+1\right) / 12$ and $c_{2}=(1-6 \lambda) / 24$. Omelyan, Mrygold and Folk[2] have computed the leading term of the shadow Hamiltonian for a large number of integration 
schemes, also including higher order and force gradient integrators. For the purpose of this discussion, however, we restrict ourselves to second order integrators. Without further knowledge of the two second order contributions to $\Delta H$, they suggest to choose $\lambda$ such that $c_{1}^{2}+c_{2}^{2}$ is minimized. As each of the two coefficients has a significantly smaller magnitude than for the leapfrog integrator (two steps of which correspond to $\lambda=1 / 4$ ), this turns out to be a sensible choice in a typical QCD setup[8], giving roughly a gain of a factor two in step-size.

\subsection{Integrator tuning}

To improve on this, Clark et al. propose to measure the values of the leading contributions to the shadow Hamiltonian as found in the actual simulation. It turns out, that its distribution does not change significantly during a trajectory, such that an equilibrium measurement on a few configurations is meaningful. An important insight gained in their work is that the size of the individual terms does not matter in the first place. This observation will also be relevant in the following section when the choice of fermion action will be discussed, where frequently the size of the fermion force (the first $\mathrm{O}\left(\delta \tau^{2}\right)$ term in Eq. 2.5) has been a leading guide in the analysis of improvements.

To understand why the size of the forces is an insufficient basis of argumentation, one first needs to notice that there are cancellations between the two terms at $\mathrm{O}\left(\delta \tau^{2}\right)$. But the size of $\Delta H$ does not matter either. What ultimately matters only is the acceptance rate in the HMC, in which only the difference $\delta H$ between the value of $H$ at the beginning and the end of the trajectory $\delta H=H_{2}-H_{1}$ enters. Since up to corrections of order $\delta \tau^{4}$ the shadow Hamiltonian $\tilde{H}$ is conserved, we can rewrite this as

$$
\delta H=\left(H_{2}-\tilde{H}_{2}\right)-\left(H_{1}-\tilde{H}_{1}\right)+\mathrm{O}\left(\delta \tau^{4}\right)=\Delta H_{1}-\Delta H_{2}+\mathrm{O}\left(\delta \tau^{4}\right) .
$$

Because the mean value $\langle\Delta H\rangle$ drops out in this difference, the fluctuations of $\Delta H$ are the quantity that determines the acceptance rate $P_{\text {acc }}$. In fact, for small energy violation the acceptance rate of the HMC is given by

$$
P_{\text {acc }}=\operatorname{erfc}\left(\sqrt{\left\langle\delta H^{2}\right\rangle / 8}\right),
$$

and therefore minimizing $\left\langle\delta H^{2}\right\rangle$ constitutes a meaningful criterion according to which algorithm can be optimized. Assuming that the fluctuations $\Delta H_{1}$ and $\Delta H_{2}$, are independent and equally distributed, this is equivalent to minimizing the variance of $\delta H$, because then[6]

$$
\left\langle\delta H^{2}\right\rangle=2 \operatorname{var}(\Delta H) .
$$

The acceptance rate is to leading order given by the variance of the difference between $H$ and the shadow Hamiltonian. The value of the norm of the forces drops completely out in this criterion. Since the variance of the norm squared gives one contribution to this improvement criterion, it might still be considered in the absence of a measurement of the second derivative of the action.

\subsection{Fermion determinant}

The strategy to evaluate integrator improvement can now be used to provide some understanding in the functioning of the determinant splitting techniques which have brought such dramatic progress towards realistic fermion simulations. 
In the standard HMC, the quark matrix determinant - here for simplicity for two degenerate flavors — is represented using a single pseudofermion[9] field $\phi$

$$
\operatorname{det} Q^{2}=\frac{1}{Z_{\phi}} \int[\mathrm{d} \phi]\left[\mathrm{d} \phi^{\dagger}\right] \exp \left\{-\left(\phi, Q^{-2} \phi\right)\right\}
$$

with $Q=\gamma_{5} D$ the massive Hermitian Dirac operator and $\phi$ complex-valued spinor fields. This identity leads to an effective one pseudofermion action $S_{1 \text { pf }}$ which is to be compared to the "exact" action $S_{\text {ex }}$, where

$$
S_{1 \mathrm{pf}}=\left(\phi, Q^{-2} \phi\right) \quad \text { and } \quad S_{\mathrm{ex}}=-\operatorname{tr} \log Q^{2} .
$$

In practice the one pseudofermion action leads to very expensive light fermion simulations[10].

Although the pseudofermion action $S_{1 \mathrm{pf}}$ for a given realization of the field $\phi$ might be very different from $S_{\mathrm{ex}}$, the force $F_{1 \mathrm{pf}}$ deriving from $S_{1 \mathrm{pf}}$ is a stochastic estimator of the force $F_{\mathrm{ex}}$ in the sense that the average over the pseudofermions of the former equals the latter

$$
\left\langle F_{1 \mathrm{pf}}\right\rangle_{\phi}=-\operatorname{tr} Q^{-2} \delta Q^{2}=F_{\mathrm{ex}} .
$$

This is at least true at the beginning of the trajectory. The fluctuations in this estimator, however, are large such that $\left|F_{1 \mathrm{pf}}\right|^{2} \gg\left|F_{\mathrm{ex}}\right|^{2}$ and

$$
\left\langle\left|F_{1 \mathrm{pf}}-\left\langle F_{1 \mathrm{pf}}\right\rangle_{\phi}\right|^{2}\right\rangle_{\phi}=\left\langle\left|F_{1 \mathrm{pf}}\right|^{2}\right\rangle_{\phi}-\left|F_{\mathrm{ex}}\right|^{2} \approx\left\langle\left|F_{1 \mathrm{pf}}\right|^{2}\right\rangle_{\phi}
$$

The large values for the norm of the fermions forces observed in practical simulations are thus a consequence of the one pseudofermion force being a poor stochastic approximation to $F_{\mathrm{ex}}$.

\subsection{Determinant factorizations}

More suitable representations of the fermion determinant can be found by splitting the contribution into several parts, each of which is introduced separately by a pseudofermion field. The physics motivation for the different methods varies, some focus on the properties of the stochastic estimator, some aim at a hierarchy of forces (in size), which then can be integrated on different time scales - possibly with the higher frequency forces being much cheaper to compute. The shadow Hamiltonian analysis can provide a framework to discuss this in a more systematic manner, and it has already become clear that just aiming at smaller forces (or equivalently a better stochastic estimate of the determinant) is not the primary target. ${ }^{1}$

Several decompositions have been proposed, the three most popular of which are mass preconditioning, the RHMC and domain decomposition. The first of those was introduced by Hasenbusch[11] and Hasenbusch and Jansen[12] where the determinant is split using a stack of (twisted) quark masses $0=\mu_{1}<\mu_{2}<\cdots<\mu_{N}$ and the identity

$$
\operatorname{det} Q^{2}=\prod_{i=1}^{N-1} \operatorname{det} \frac{Q^{2}+\mu_{i}^{2}}{Q^{2}+\mu_{i+1}^{2}} \times \operatorname{det}\left(Q^{2}+\mu_{N}^{2}\right),
$$

\footnotetext{
${ }^{1}$ Smaller forces can still be beneficial as they help avoid problems in connection with the stability of the MD integration.
} 


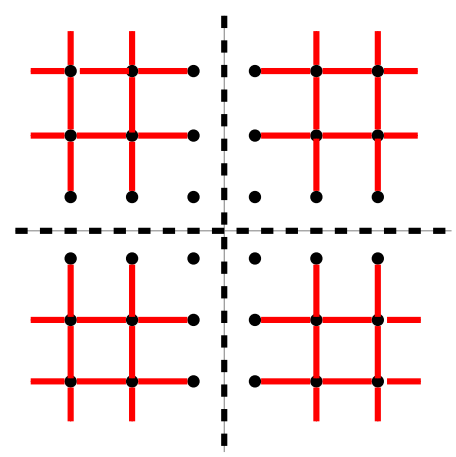

Figure 1: Decomposition of the Dirac operator in the DD-HMC algorithm. The solid lines indicate the gauge links contributing to $Q_{\Lambda}$.

where each determinant is represented by a single pseudofermion field. ${ }^{2}$ These masses can be tuned, a choice which will also influence the relative cost of their evaluation[13].

Alternatively, the RHMC[14] decomposes the determinant into equal factors using the $N$-th root of the fermion matrix, which needs to be implemented by a rational approximation

$$
\operatorname{det} Q^{2}=\prod_{i=1}^{N} \operatorname{det} \sqrt[N]{Q^{2}}
$$

Each of these factors is again represented by a pseudofermion. The RHMC is primarily used for simulating single flavors, but is also employed for pairs of degenerate quarks as in Eq. 2.14. The evaluation of the rational approximation, however, is quite costly, in particular because the frequently employed multi-shift solvers do not combine well with the inexact preconditioning techniques used for light fermions.

Finally the DD-HMC algorithm[15] is based on a geometrical block decomposition, where the Dirac operator restricted to the blocks $Q_{\Lambda}$ is considered in one factor

$$
\operatorname{det} Q^{2}=\prod_{\Lambda} \operatorname{det} Q_{\Lambda}^{2} \times \operatorname{det} Q_{S}^{2},
$$

and the second factor is a matrix which has the same determinant as the Schur complement of $Q$ with respect to the block projection. This algorithm as been successfully used in many Wilson fermion simulations, however, it suffers from the links between the blocks which do not get updated during a trajectory, which causes increased autocorrelation times[16, 17].

A detailed comparison between these algorithms for light quark simulations has not been published. Just because of its simplicity, we now study the effect of the quark determinant splitting at the example of the Hasenbusch decomposition.

\subsection{Numerical examples}

The numerical examples in these proceedings come from a simulation described in detail in Ref. [18]. In particular we use a run with $2+1$ dynamical flavors of non-perturbatively improved

\footnotetext{
${ }^{2}$ There are many versions of this splitting, with shifts in the mass, the twisted mass and also applying the factorization in Eq. 2.13 to the Schur complement in even-odd preconditioning. This version is chosen for ease of notation.
} 

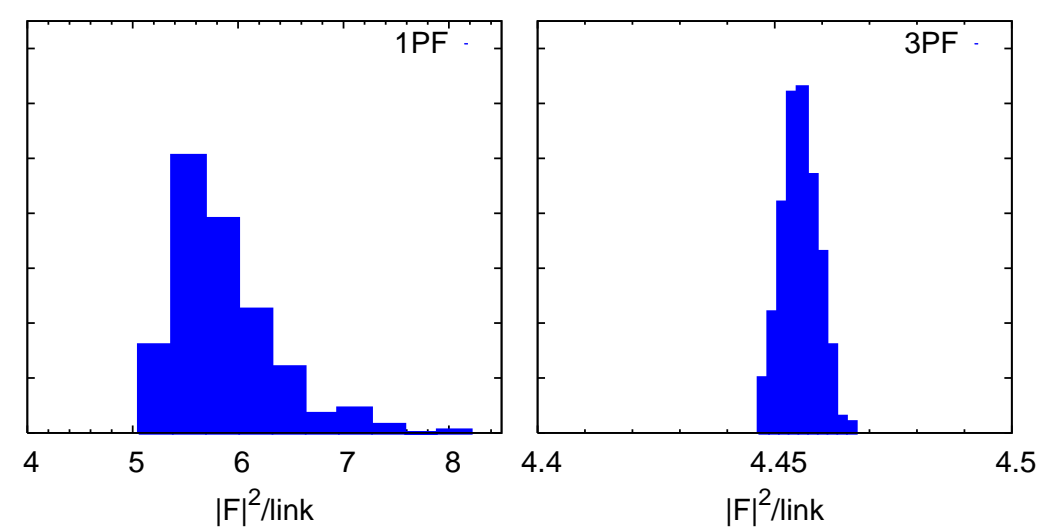

Figure 2: Distribution of the norm of the force with the one pseudofermion action and using three pseudofermions in the Hasenbusch splitting. The size of the forces is only marginally reduced, but the fluctuations of the norm of the force decrease drastically. Note the different scale of the $x$-axes.

Wilson fermions and the Iwasaki gauge action. This setup has been extensively studied by the PACS-CS collaboration[19, 20]. The pion mass is $200 \mathrm{MeV}$ and strange quark mass is at physical value, which means that the $64 \times 32^{3}$ lattices at a lattice spacing of about $a=0.09 \mathrm{fm}$ have $L \approx$ $3.1 \mathrm{fm}$ and $m_{\pi} L \approx 3.1$.

In this simulation, which has been performed with the publicly available openQCD code, ${ }^{3}$ open boundary conditions in time have been used together with twisted mass reweighting, which will both be described below. The three twisted masses in the frequency splitting, applied on the Schur complement of the even-odd preconditioning, have been chosen roughly equally spaced on a log-scale. The strange quark is simulated with the RHMC algorithm. Although some details will be different, the main statements are expected to carry over to different gauge and fermion discretizations.

\subsection{Effect of the Hasenbusch decomposition}

To illustrate the impact of the Hasenbusch mass splitting, we have measured the first and second derivative of the action on the ensemble introduced in the previous section, using 10 gauge configurations and 30 realizations of the pseudofermion fields on each. In Fig. 2, the distribution of the norm squared of the fermion force is shown. In the left panel just one pseudofermion field is seen to lead to large fluctuations in the norm of the force. By using the Hasenbusch splitting with $N=3$, these fluctuations are drastically reduced. Note that the norm of the force itself is not very much smaller, i.e., the fluctuation of the force remains large; mass preconditioning is doing little towards getting a better stochastic estimate of the force.

However, as we have seen in the previous section, this is not needed. Smaller fluctuations of the norm of the force are already more significant, but what actually counts is the variance of the higher order terms in the shadow Hamiltonian $\Delta H$ that enters the acceptance rate Eq. 2.7. The

\footnotetext{
${ }^{3}$ The code is available under http://cern. ch/luscher/openQCD.
} 

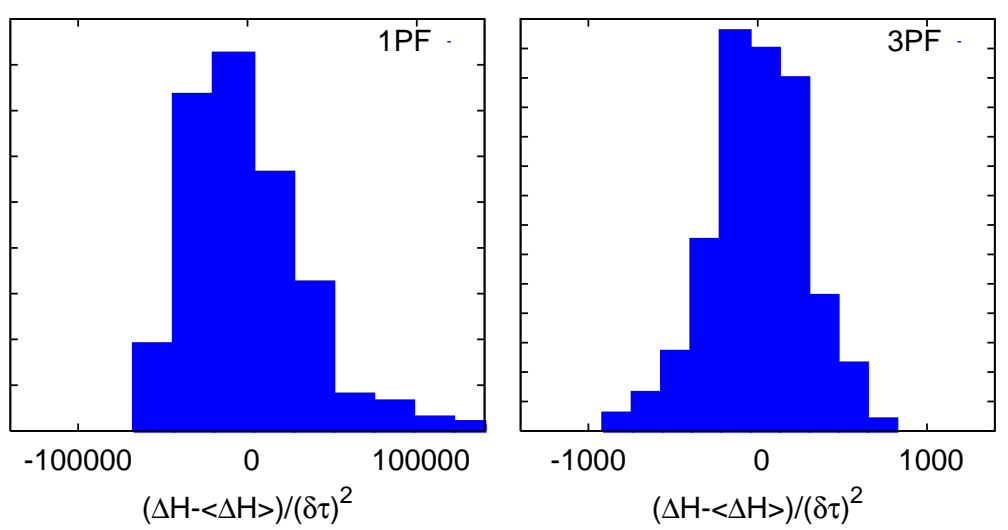

Figure 3: Fluctuation of the value of the shadow Hamiltonian around its mean value, fermionic contribution only. Left panel the one pseudofermion action, right panel using three pseudofermions in the Hasenbusch splitting.

width of its distribution shown in Fig. 3 is drastically reduced by the two additional pseudofermion fields. To achieve the same acceptance rate, this allows for an order of magnitude larger step size and a correspondingly cheaper simulation.

The discussion in this section just wants to illustrate the functioning of the determinant splitup. Also domain decomposition and the RHMC are successfully used in modern dynamical fermion simulations, which hints on a similar effect on the distribution of $\Delta H$ for these algorithms.

\subsection{Multiple time scales}

The size of the gauge and the many components of the fermion force frequently varies over many orders of magnitude. This has lead to the suggestion to integrate them on different time scales[3], which is particularly natural if the larger forces are also much cheaper to compute. The gauge forces, e.g., have typically a much larger norm and require significantly less computer time than the fermion forces. Technically, if the total action is split in two parts $S=S_{1}+S_{2}$, one integrates one component of the action $S_{1}$ with the integrator in eq. 2.4, but instead of $T_{\pi}$, one puts $m$ steps of eq. 2.4, where only the force corresponding to $S_{2}$ is applied.

Also the split determinants have been combined with multiple time scales[21, 13]. From the shadow Hamiltonian it can be understood why large hierarchies in the size of the forces do not necessarily translate to corresponding hierarchies in the time steps. The shadow Hamiltonian for the multiple time scale integrator reads[4]

$$
\begin{aligned}
\widetilde{H}= & H+\delta \tau^{2}\left\{c_{1} \sum_{x, \mu} \partial_{x, \mu}^{a} S_{1} \partial_{x, \mu}^{a} S_{1}-c_{2} \sum_{\substack{x, \mu \\
y, v}} \pi_{x, \mu}^{a} \pi_{y, v}^{b} \partial_{x, \mu}^{a} \partial_{y, v}^{b} S_{1}+c_{2} \partial_{x, \mu}^{a} S_{1} \partial_{x, \mu}^{a} S_{2}\right. \\
& \left.\frac{1}{m^{2}}\left(c_{1} \sum_{x, \mu} \partial_{x, \mu}^{a} S_{2} \partial_{x, \mu}^{a} S_{2}-c_{2} \sum_{\substack{x, \mu \\
y, v}} \pi_{x, \mu}^{a} \pi_{y, v}^{b} \partial_{x, \mu}^{a} \partial_{y, v}^{b} S_{2}\right)\right\}+\ldots,
\end{aligned}
$$

where the term in the second row reflects the hierarchy of the integration steps. The third term in the first row containing the interference between the forces from $S_{1}$ and $S_{2}$, however, is not suppressed 
by $1 / \mathrm{m}^{2}$ even though it depends on $S_{2}$. Since in typical settings the forces deriving from $S_{2}$ are much larger than those from $S_{1}$, this term can give a large contribution.

Obviously, all this depends on the covariance of the various force contributions and a complete picture needs detailed measurements of these terms. The smaller the covariance between the two forces, the better the multiple time scale method will work, but in particular for the various components of the fermion force, large covariances are to be expected. In any case, if the forces from $S_{2}$ dominate, or are much cheaper to compute, the multiple time scales can be beneficial[13].

To suppress this contribution from the interference term, the coefficient $c_{2}$ can be tuned to zero by choosing $\lambda=1 / 6$. Although this is not a good integrator for the forces deriving from $S_{1}-$ it does not profit from the cancellation between the two contributions in the shadow Hamiltonian this can be a good choice if these forces and their fluctuations are small.

\subsection{Stability of Wilson fermion simulations}

Large forces are known to cause the numerical integrators to become unstable above a certain step size. Here light Wilson fermions have a particular problem due to the lack of a spectral gap, which results in potentially unbounded forces where eigenvalues become zero. The most obvious consequence of this are the so-called "spikes", exceptionally large values of $\delta H$, which are observed during the simulation. Furthermore, the molecular dynamics trajectories cannot cross the surfaces of zero eigenvalues, at least if the integration is exact. This means that the simulation is not ergodic and ergodicity depends on integration errors. Also thermalization can be affected by the evolution being stuck in one of these sectors.

Introducing a small twisted mass during the simulation into the light Dirac operator can cure these problems and it has been argued in Ref. [22] that even on typical large volumes its effect can be reliably cancelled by including a corresponding reweighting term into the measurement. Two possible replacements for the fermion determinant have been proposed

$$
\operatorname{det} Q^{2} \rightarrow \begin{cases}\operatorname{det}\left(Q^{2}+\mu^{2}\right) & \text { Type I } \\ \operatorname{det}\left(Q^{2}+\mu^{2}\right)^{2} / \operatorname{det}\left(Q^{2}+2 \mu^{2}\right) & \text { Type II, }\end{cases}
$$

where the second option has the advantage that the contribution from large eigenvalues $\lambda$ of $Q$ to the reweighting factor $R$-corresponding to the ratio of $\operatorname{det} Q^{2}$ to what it has been replaced with - is suppressed by $\mu^{4} / \lambda^{4}$, whereas the first falls of with $\mu^{2} / \lambda^{2}$. Since large contributions in the ultraviolet limit the reach in $\mu$ of the reweighting, and therefore the benfits that can be achieved in the infrared, the second choice is likely to yield better results.

For the simulation described in Sec. 2.5, the second option has been employed, with $\mu$ large enough to efficiently suppress large values of $|\delta H|$. In fact, on at most a few per-mille of the trajectories it assumed values above 2. Still, as can be seen from the measured reweighting factors in Fig. 4, its fluctuations are under control and only $15 \%$ of the configurations obtain a weight below 0.5 .

Also reweighting in the quark mass (instead of a twisted mass term) has been proposed[23] and makes part of what is used in large scale simulations[19, 20,24]. Here the emphasis is less on a strict spectral bound but on possible corrections in the tuning or the possibility to reach smaller quark masses more cheaply. In both reweighting methods, the over-sampling of small eigenvalues can also lead to reduced fluctuations in some observables, e.g., the pion correlator. 


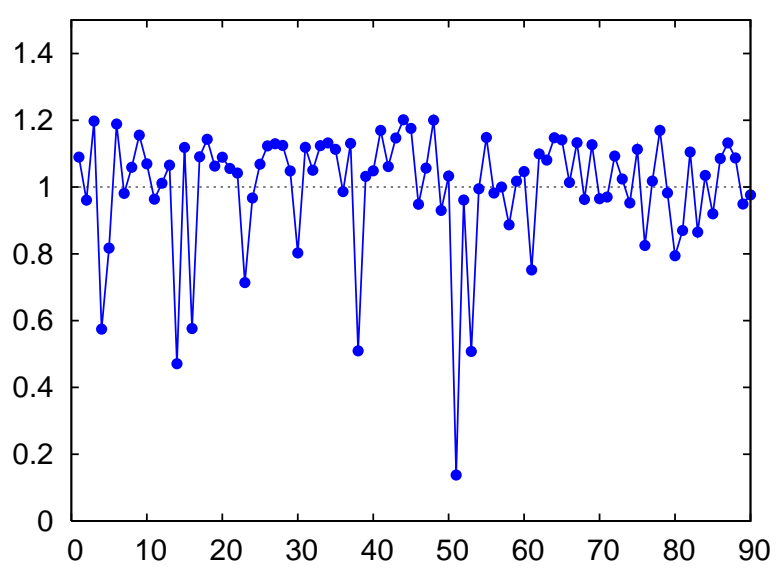

Figure 4: Reweighting factor $R$ for the second type of reweighting in the simulation described in the text.

\subsection{Solver}

A main difficulty of going towards smaller quark masses has been the rise of the condition number of the fermion matrix as $a m_{q} \rightarrow 0$ and the associated increase in iteration count of the iterative solvers. Deflation techniques which remove the contribution of the low-modes from the linear system substantially alter this situation. Explicitly computing (an approximation to) the $N$ lowest eigenmodes, however, is not an option in large volume simulations, since $N$ needs to be scaled with $V$ in order to keep the condition number approximately constant.

This has been changed by the advent of local deflation techniques[25], where the space which is projected from the system is spanned by local (block) modes. The number of these modes per block is kept fixed as the volume is increased, which means that the dimension of the space spanned is growing with the volume. The resulting iterative, locally deflated solver shows only a very modest, if any, increase in iteration count as the quark is lowered towards the chiral limit. Closely related to this approach are adaptive multigrid algorithms[26,27] which consequently show similar performance gains and are also being developed for use together with domain wall fermions[28].

All these techniques require a certain amount of setup time and also the memory usage can be substantial. This is, however, easily amortized as the force evaluation in the determinant splitting techniques need the solution of the Dirac equation for many right hand sides on the same gauge field.

\section{Continuum limit}

Approaching the continuum limit is an essential part of a field theory calculation, but in most practical simulations the lattice spacing can only be varied by a modest factor (and still lie within the scaling regime). This is due to the very steep increase in computational cost as the continuum limit is approached if the physical volume is to be held fixed. The number of lattice points increases with $a^{-4}$ and to get fixed acceptance rate with a second order integrator requires $a^{-1}$ steps per trajectory. Furthermore, Monte Carlo time itself scales with the lattice spacing and autocorrelation 
times of observables $A$ are expected to show a universal behavior

$$
\tau_{\text {int }}(A) \propto a^{-z}
$$

with a dynamical critical exponent $z$, which for the HMC algorithm is $z \approx 2$ as argued in Sec. 3.1. The total cost is, thus, expected to rise with the seventh power of the inverse lattice spacing, each factor of two in $a$ translating into more than two orders of magnitude in cost. There might be some effects from reduced noise due to smoother gauge fields, but it is still necessary to produce a Monte Carlo time history which contains at least $\mathrm{O}(100)$ times the largest exponential autocorrelation time, setting a lower bound for the numerical effort, irrespective of the desired accuracy.

In the traditional setup of QCD simulations using periodic boundary conditions for the gauge fields, an additional problem arises due to the global topological charge. Field space in the continuum is disconnected and decomposed into sectors of different integer topological charge,

$$
Q=\frac{1}{32 \pi^{2}} \int \mathrm{d} x \varepsilon_{\mu \nu \rho \sigma} F_{\mu \nu} F_{\rho \sigma} .
$$

We therefore expect that close to the continuum limit any algorithm which changes the fields (quasi)-continuously has difficulty changing the global topological charge of the gauge configuration.

How quickly this continuum behavior is approached is a priori not clear and details will in general depend on the gauge and fermion discretizations. However, pure gauge theory with the Wilson gauge action has exhibited a drastic rise in the autocorrelation time of $Q^{2}$, compatible with $\tau_{\text {int }}\left(Q^{2}\right) \propto a^{-5}$, see Fig. 5, but could also be exponential in $a^{-1}[29]$.

In a 2010 contribution to this conference series[30], Lüscher observed that in pure gauge theory, in a fixed volume the probability to find a configuration which has a large plaquette value and is therefore in between topological sectors (plaquettes constructed from links smoothed by the Wilson flow) decreases as $a^{-6}$ as the continuum limit is approached and the volume is kept fixed. This indicates the rapid formation of topological sectors and corresponds to the drastic rise in $\tau_{\text {int }}\left(Q^{2}\right)$ as $a \rightarrow 0$.

The situation with dynamical fermions has been discussed at this conference. For the ETM collaboration, Deuzeman[32] reported an absence of slowing down of the topological charge towards the continuum, whereas in the Wilson fermion simulations with the DD-HMC algorithm Mondal found a substantial increase in autocorrelations[33]. An exceptionally slow evolution of the topological charge in the presence of dynamical fermions has in the past also been found for a variety of gauge and fermion discretizations [34, 35, 36, 37].

Let us briefly note that the problem of a slow global topological charge could in principle be solved by fixing the topological sector during the simulation[38]. With a different motivation, the JLQCD collaboration has been using this for their dynamical overlap simulations[39]. In this setup one has to deal with power-like finite volume corrections that vanish only as $V^{-1}$ [40], and whose analytic form is not known for all observables. The impact of the fixed topological charge on the algorithmic performance has so far not been studied close to the continuum limit.

\subsection{Expected scaling}

The question remains whether the observed scaling for the topological charge is exceptional, or whether there is reason to expect that autocorrelations should rise with a smaller power of the 


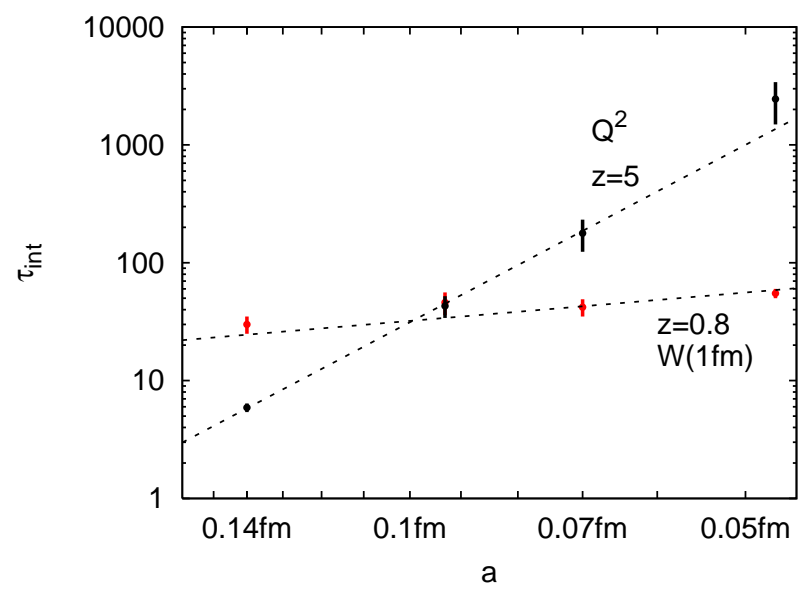

Figure 5: Integrated autocorrelation time vs. lattice spacing for a smeared Wilson loop of size $(1 \mathrm{fm})^{2}$ and the topological charge in pure gauge theory with Wilson gauge action. Data from Ref. [31].

inverse lattice spacing. From dimensional analysis of the HMC equations of motion autocorrelation times should scale with the inverse lattice spacing[41], once the trajectory length is scaled accordingly. This does not match with the experience form measurements as in Fig. 5.

This free field theory result, however, cannot be expected to hold in the interacting theory as has been argued in Ref. [42]. There, an attempt to prove the renormalizability of the fivedimensional field theory - defined by the four-dimensional physical field theory augmented by the dynamics in simulation time as a fifth dimension — has lead to a divergence that could not be removed by a local counter term.

These methods have earlier been used to prove the renormalizability of the Langevin equation for scalar field theory and gauge theory[43, 44]. This means that up to logarithmic corrections, the free field result of $z=2$ holds for algorithms based on this stochastic differential equation. In Ref. [42] it has been conjectured that the HMC algorithm falls into the universality class of the Langevin equation. For observables $A$ with a proper continuum limit, it is therefore expected that the integrated autocorrelation function scales with the inverse lattice spacing squared

$$
\tau_{\text {int }}(A, W) \propto a^{-2} \quad \text { with } \quad \tau_{\text {int }}(A, W)=\frac{1}{2} \tau+\tau \sum_{k=1}^{W} \frac{\Gamma_{A}(k \tau)}{\Gamma_{A}(0)}
$$

and $\Gamma=\langle(A(t)-\bar{A})(A(0)-\bar{A})\rangle$ the autocorrelation function and $\tau$ the distance between two measurements.

These arguments involve a continuum limit of the autocorrelation function and the question for the algorithmic analysis is whether the limits $a \rightarrow 0$ and $W \rightarrow \infty$ commute. For the performance of the algorithms, one is interested at the $W \rightarrow \infty$ limit at fixed lattice spacing, whereas the theoretical arguments apply to taking the continuum limit first. But in general, a quadratic scaling should be expected also for $\tau_{\text {int }}$ and the scaling found in the topological charge can indeed be considered to be exceptionally strong.

An illustration of these statements can be found in data from the $\mathrm{CP}^{9}$ model[45] presented in Fig. 6. For an earlier discussion of topological slowing down in this model see Ref. [29]. The 


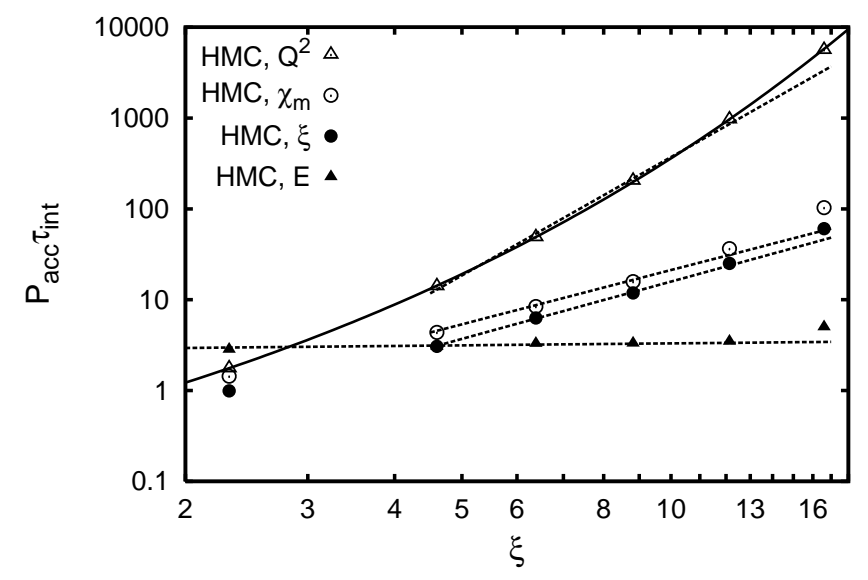

Figure 6: Scaling of the integrated autocorrelation times with the lattice spacing in the $\mathrm{CP}^{9}$ model[45]. The topological charge squared shows a behavior compatible with an exponential growth indicated by the solid line, the dashed line follows a power law with $z=4$. For the magnetic susceptibility $\chi_{m}$ and correlation length $\xi$ the dashed lines show a scaling with $\xi^{2}$, whereas the corresponding curve for the action density $E$ has $z=0.12$.

magnetic susceptibility $\chi_{m}$ and the correlation length $\xi$ show a scaling of $\tau_{\text {int }}$ compatible with a dynamical critical exponent $z=2$. They both have a meaningful continuum limit. This is not the case for the action density $E$, which exhibits an almost constant behavior. However, the topological charge shows a growth in the autocorrelation time which is compatible with an exponential behavior, as already proposed in Ref. [29]. At a certain point, the dependence of the other observables on the topological charge - small as it may be - dominates all autocorrelation functions and causes a deviation from the previous behavior. This also exemplifies the danger the frozen charge poses for simulations in QCD, where a seemingly decoupled observable can suddenly receive important contribution to its autocorrelations from the slow modes.

\subsection{Open boundary conditions}

Since we have argued that exceptionally long autocorrelations of the topological charge are linked to the topological sectors in the continuum, a setup in which these sectors do not exist is likely to solve the problem. In Ref. [46] it has been proposed to use open boundary conditions in time, such that the topological charge can continuously change over these boundaries. Field space in the continuum is no longer disconnected; there is no integer topological charge. In the spatial directions, periodic boundary conditions are kept. Technically, this means to impose for the quark and antiquark fields $\psi(x)$ and $\bar{\psi}(x)$,

$$
\begin{array}{ll}
\left.P_{+} \psi(x)\right|_{x_{0}=0}=\left.P_{-} \psi(x)\right|_{x_{0}=T}=0, & P_{ \pm}=\frac{1}{2}\left(1 \pm \gamma_{0}\right), \\
\left.\bar{\psi}(x) P_{-}\right|_{x_{0}=0}=\left.\bar{\psi}(x) P_{+}\right|_{x_{0}=T}=0, &
\end{array}
$$

like in the Schrödinger functional. For the gauge fields

$$
\left.F_{0 k}(x)\right|_{x_{0}=0}=\left.F_{0 k}(x)\right|_{x_{0}=T}=0 \quad \text { for all } \quad k=1,2,3
$$


is chosen.

Since periodic boundary conditions in the spatial directions are imposed, the projection to definite momentum of the operators is still possible and the impact on the physics analysis is minimal, because in the time direction the transfer matrix of the theory is not changed. The boundary conditions will be reflected in the hadronic correlators, but the particle spectrum will be the same.

\subsection{Observables}

To ensure that a simulation is reliable, it is pivotal to have a Monte Carlo history that is much longer than the longest exponential autocorrelation time. To this end it is not sufficient to examine the autocorrelation function of the observable in question, because in a noisy observable the exponential tail in the autocorrelation function can easily be covered by statistical fluctuations; a seemingly small $\tau_{\text {int }}(A)$ can be a consequence of a short run history. For the arguments concerning the renormalizability of the algorithm to apply, it is also beneficial to consider quantities with a well defined continuum limit

Such observables, which also have low statistical noise, can be constructed using the Wilson flow $[47,48,49]$, defined by a partial differential equation on the gauge fields

$$
\partial_{t} V_{t}(x, \mu)=-a g_{0}^{2} T^{a}\left(\partial_{x, \mu}^{a} S_{\mathrm{W}}\right)\left(V_{t}\right) V_{t}(x, \mu),\left.\quad V_{t}(x, \mu)\right|_{t=0}=U(x, \mu) .
$$

Starting from the gauge fields $U(x, \mu)$, integrating this equation up to a flow time $t$ defines gauge fields $V(x, \mu)$ that are smoothed out over a radius $r=\sqrt{8 t}$.

In the following, we will consider three such observables, the action density and the topological charge density summed over a time slice as well as the global topological charge

$$
\begin{array}{ll}
\bar{Q}\left(x_{0}\right)=-\frac{a^{3}}{32 \pi^{2}} \sum_{\vec{x}} \varepsilon_{\mu v \rho \sigma} \operatorname{tr}\left\{G_{\mu v}(x) G_{\rho \sigma}(x)\right\} ; \quad Q=a \sum_{x_{0}} \bar{Q}\left(x_{0}\right) ; \\
\bar{E}\left(x_{0}\right)=-\frac{a^{3}}{2 L^{3}} \sum_{\vec{x}} \operatorname{tr}\left\{G_{\mu \nu}(x) G_{\mu v}(x)\right\} .
\end{array}
$$

For the sliced observables, the central time slice will be considered. The crucial point of these observables is that the smoothing radius is kept fixed as the continuum limit is approached. In the following, the flow time will be taken to at $t_{0}$, i.e., the flow time at which $\left.t^{2}\langle E\rangle\right|_{t=t_{0}}=0.3$ intoduced in Ref. [48], which corresponds to a smoothing radius of $\sqrt{8 t_{0}} \approx 0.42 \mathrm{fm}[50]$.

The smoothing has a notable effect on the integrated autocorrelation time, as is demonstrated in Fig. 7 taken from Ref. [46] for pure gauge theory and a lattice spacing of $a \approx 0.05 \mathrm{fm}$. The integrated autocorrelation time $\tau_{\text {int }}(\bar{E})$ increases by roughly an order of magnitude, highlighting the importance of choosing smooth quantities with little noise. The behavior is well described by an exponential approach to a constant $\tau_{\text {int }}(\bar{E})=a+b \exp (-t / c)$, with the autocorrelations at $t=t_{0}$ being close to saturation.

\subsection{Effect of the open boundary conditions on autocorrelations}

The open boundary conditions in time have been studied in pure gauge theory with the Wilson action in Ref. [46] from which Fig. 8 has been taken. It shows the scaling of the integrated autocorrelation time with the lattice spacing on a lattice of constant physical volume $V=(1.6 \mathrm{fm})^{4}$. 


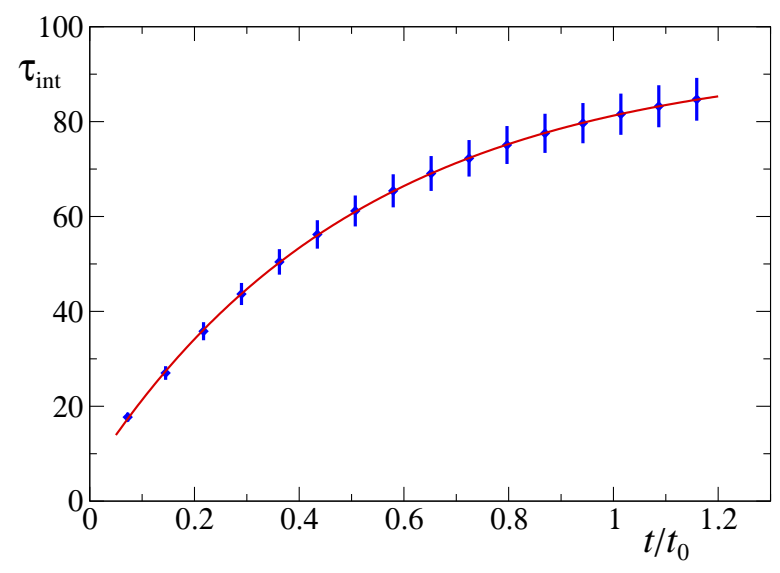

Figure 7: Integrated autocorrelation time of $\bar{E}$ as a function of the flow time in units of $t_{0}$. The solid line shows a fit to the data of the functional dependence discussed in the text.

Results for the stochastic molecular dynamics algorithm(SMD)[51] and the HMC algorithm are shown. The two data sets show the same scaling behavior and differ only by a global normalization factor.

All three observables follow the $a^{-2}$ expectation, no sign of topological freezing could be found, even though the scale covers roughly the same range of lattice spacings as in Fig. 5, which was using the same gauge action with periodic boundary conditions. The autocorrelation time of the topological charge is significantly reduced by the open boundaries. For the HMC at $a \approx 0.05 \mathrm{fm}$, we have $\tau_{\text {int }}\left(Q^{2}\right) \approx 1000$ with the periodic boundary conditions and $\tau_{\text {int }}\left(Q^{2}\right) \approx 180$ with open boundaries. Remarkably, the autocorrelation times of the topological quantities and for $\bar{E}$ are not very different.

\subsection{Effect on hadron correlation functions}

Since the open boundary conditions work as expected for the autocorrelations, we now have to study their effect on physical observables. On general grounds, one expects their effect to be exponentially suppressed by the distance from $x_{0}=0$ and $x_{0}=T$. Closer to the boundaries, of course, the boundary conditions are reflected in the temporal dependence of the correlators.

The (light) pseudoscalar correlation function is known to be dominated by pions in the long distance and light quark mass regime. Since Dirichlet boundary conditions are generic in scalar field theories, it is therefore natural to assume that the pion filed $\pi^{a}$ vanishes on the boundaries

$$
\left.\pi^{a}(x)\right|_{x_{0}=0}=\left.\pi^{a}(x)\right|_{x_{0}=T}=0 .
$$

For the pion propagator $G_{\pi}\left(x_{0}, y_{0}\right)$, one infers by solving the Klein-Gordon equation that for $x_{0}>y_{0}$ it assumes in the region of $x_{0}$ not too close to the boundaries the form

$$
G_{\pi}\left(x_{0}, y_{0}\right) \propto \sinh \left(m_{\pi}\left(T-x_{0}\right)\right) .
$$

This is confirmed by the measured data shown in Fig. 9, where a fit of Eq. 3.9 to the data with sufficient distance from the boundaries is shown. 


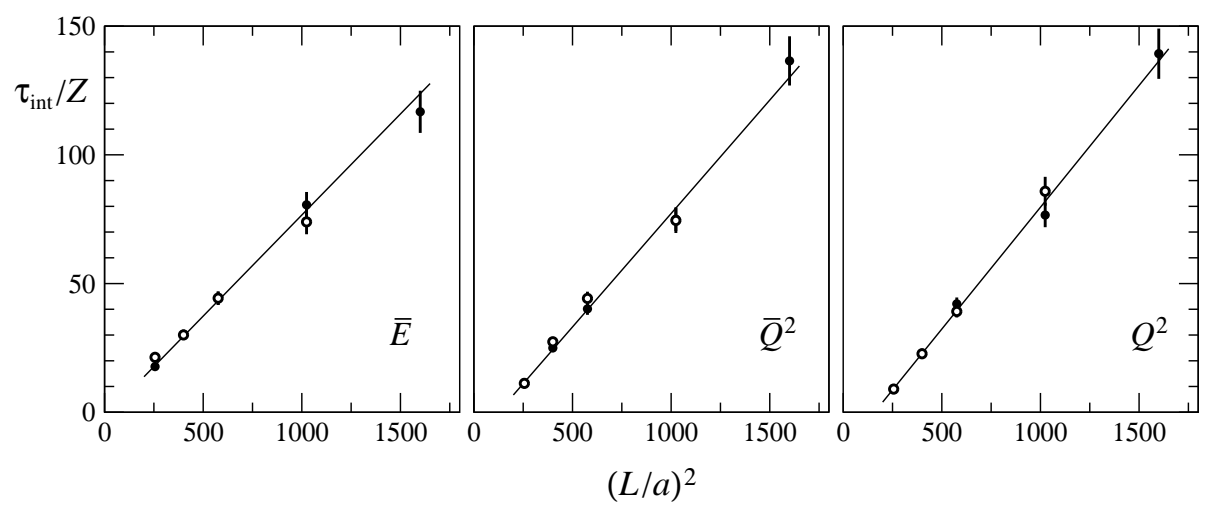

Figure 8: Scaling of the integrated autocorrelation times in pure gauge theory with open boundary conditions in time towards the continuum limit. The observed scaling matches with a dynamical critical exponent of $z=2$. The topological charge does not show exceptional slowing down. Filled symbols are results for the SMD algorithm with $Z=1$, open symbols for the HMC $Z=1.32$.

As can be seen from this example, the analysis changes due to the new boundary conditions, but not in a dramatic way. Experience with more complicated correlation functions will need to be gained in order to have a more complete picture of their consequences.

\section{Conclusions}

Few of the methods discussed in this contribution are new, still recent years have witnessed substantial progress in our ability to simulate QCD with light quarks on fine lattices. This is mainly due to an improved understanding of these methods and synergies gained from combining them.

The determinant splitting techniques allow for much larger step sizes, which can only be understood using the theory of symplectic integrators. The determinant splitting, along with techniques like twisted mass reweighting, significantly reduce fluctuations in the forces and in turn make higher order and force gradient integrators proposed during the last decade profitable. And also the sophisticated solvers for the Dirac equation have their setup cost easily amortized over the many solutions needed in each individual step. Each of these components individually already brings some gain, but it is in their combination that the full potential can be reached. And there might be additional gains from better further understanding possible.

In the traditional setup, lattice QCD simulations are performed with periodic boundary conditions, where the freezing of the topological charge causes a severe problem with ergodicity as the continuum limit is approached. With typical resources, simulations with a lattice spacing below $0.05 \mathrm{fm}$ are not possible, and the problem is sufficiently generic that an algorithmic solution seems unlikely in the near future. Changing the lattice setup by using open boundary conditions, however, solves the issue.

Still, QCD simulations are expensive. To keep finite volume effects under control, the size of the box needs to be scaled with the inverse pion mass and physical pions require a box size of about $6 \mathrm{fm}$. Fine lattice spacings are currently difficult to reach with such a volume. 


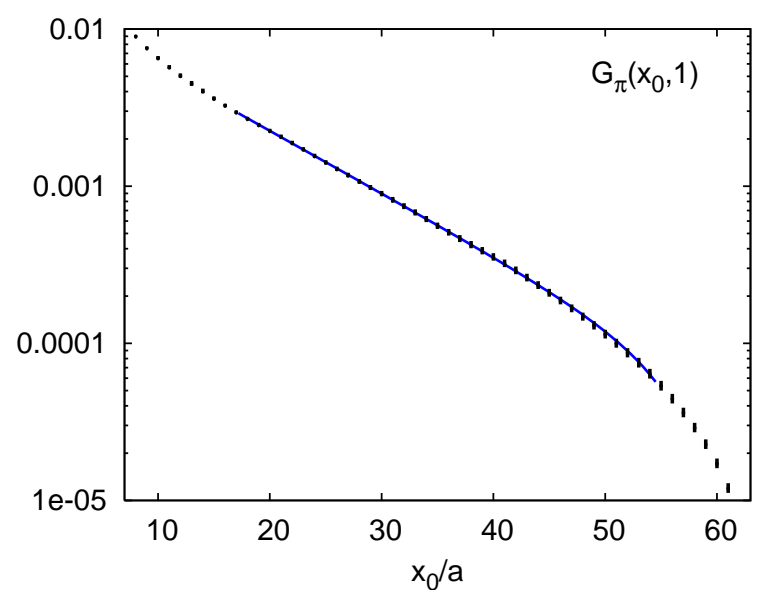

Figure 9: Pseudoscalar correlation function with the source at $x_{0}=a$. A fit to the chiral perturbation theory formula is shown, which supports the hypothesis of Dirichlet boundary conditions for the pion fields.

From the simulations as described in Sec. 2.5 but at the physical pion mass, we can now estimate the order of magnitude of the computer resources needed to repeat the simulation on a sufficiently large volume with spatial extent $L=6 \mathrm{fm}$ and a finer lattice. At $a=0.09 \mathrm{fm}$, we estimate $\tau_{\text {int }}(\bar{E}) \sim 20$ and we assume that this is a one of the slowest observables. If we want a run length of at least $100 \times \tau_{\text {int }}(\bar{E})$, we arrive at a cost estimate of such a simulation

$$
C=3 \text { Tflops } \times \text { years } \times\left(\frac{a}{0.09 \mathrm{fm}}\right)^{-7} .
$$

This means that a $a=0.045 \mathrm{fm}$ lattice still requires 400 Tflops $\times$ years. Hopefully, further progress will reduce this number.

\section{Acknowledgments}

It is a pleasure to thank G. Engel, M. Lüscher, M. Marinkovic, R. Sommer and F. Virotta for collaboration and many discussions on issues presented here. Also correspondence with B. Leder and $\mathrm{U}$. Wenger is thankfully acknowledged.

\section{References}

[1] S. Duane, A. D. Kennedy, B. J. Pendleton, and D. Roweth, Hybrid Monte Carlo, Phys. Lett. B195 (1987) 216.

[2] I. P. Omelyan, I. M. Mryglod, and R. Folk, Symplectic analytically integrable decomposition algorithms: classification, derivation, and application to molecular dynamics, quantum and celestial mechanics simulations, Computer Physics Communications 151 (2003), no. 3272 - 314.

[3] J. C. Sexton and D. H. Weingarten, Hamiltonian evolution for the hybrid Monte Carlo algorithm, Nucl. Phys. B380 (1992) 665-678.

[4] M. Clark, A. Kennedy, and P. Silva, Tuning HMC using Poisson brackets, PoS LATTICE2008 (2008) 041, [arXiv:0810.1315]. 
[5] M. Clark, B. Joo, A. Kennedy, and P. Silva, Better HMC integrators for dynamical simulations, PoS LATTICE2010 (2010) 323, [arXiv: 1011 . 0230].

[6] M. Clark, B. Joo, A. Kennedy, and P. Silva, Improving dynamical lattice QCD simulations through integrator tuning using Poisson brackets and a force-gradient integrator, Phys.Rev. D84 (2011) 071502, [arXiv:1108.1828].

[7] A. Kennedy, P. Silva, and M. Clark, Shadow Hamiltonians, Poisson Brackets, and Gauge Theories, arXiv: 1210.6600 .

[8] T. Takaishi and P. de Forcrand, Testing and tuning new symplectic integrators for hybrid Monte Carlo algorithm in lattice QCD, Phys.Rev. E73 (2006) 036706, [hep-lat / 0505020 ].

[9] D. Weingarten and D. Petcher, Monte Carlo Integration for Lattice Gauge Theories with Fermions, Phys.Lett. B99 (1981) 333.

[10] CP-PACS and JLQCD Collaboration, A. Ukawa, Computational cost of full QCD simulations experienced by CP-PACS and JLQCD Collaborations, Nucl.Phys.Proc.Suppl. 106 (2002) 195-196.

[11] M. Hasenbusch, Speeding up the Hybrid-Monte-Carlo algorithm for dynamical fermions, Phys. Lett. B519 (2001) 177-182, [hep-lat/ 0107019 ].

[12] M. Hasenbusch and K. Jansen, Speeding up lattice QCD simulations with clover-improved Wilson fermions, Nucl. Phys. B659 (2003) 299-320, [hep-lat/0211042].

[13] C. Urbach, K. Jansen, A. Shindler, and U. Wenger, HMC algorithm with multiple time scale integration and mass preconditioning, Comput. Phys. Commun. 174 (2006) 87-98, [hep-lat/0506011].

[14] M. Clark and A. Kennedy, Accelerating dynamical fermion computations using the rational hybrid Monte Carlo (RHMC) algorithm with multiple pseudofermion fields, Phys.Rev.Lett. 98 (2007) 051601, [hep-lat/0608015].

[15] M. Lüscher, Schwarz-preconditioned HMC algorithm for two-flavour lattice QCD, Comput. Phys. Commun. 165 (2005) 199, [hep-lat/ 0409106 ].

[16] S. Schaefer, R. Sommer, and F. Virotta, Investigating the critical slowing down of QCD simulations, PoS LAT2009 (2009) 032, [arXiv: 0910 . 1465].

[17] M. Marinkovic and S. Schaefer, Comparison of the mass preconditioned HMC and the DD-HMC algorithm for two-flavour QCD, PoS LATTICE2010 (2010) 031, [arXiv: 1011.0911 ].

[18] M. Lüscher and S. Schaefer, Lattice QCD with open boundary conditions and twisted-mass reweighting, arXiv:1206.2809.

[19] PACS-CS Collaboration, S. Aoki et al., 2+1 Flavor Lattice QCD toward the Physical Point, Phys.Rev. D79 (2009) 034503, [arXiv:0 807.1661 ].

[20] PACS-CS Collaboration, S. Aoki et al., Physical Point Simulation in 2+1 Flavor Lattice QCD, Phys.Rev. D81 (2010) 074503, [arXiv:0911.2561].

[21] QCDSF Collaboration Collaboration, A. Ali Khan et al., Accelerating the hybrid Monte Carlo algorithm, Phys.Lett. B564 (2003) 235-240, [hep-lat/ 0303026 ].

[22] M. Lüscher and F. Palombi, Fluctuations and reweighting of the quark determinant on large lattices, PoS LATTICE2008 (2008) 049, [arXiv: 0810 . 0946].

[23] A. Hasenfratz, R. Hoffmann, and S. Schaefer, Reweighting towards the chiral limit, Phys.Rev. D78 (2008) 014515, [arXiv:0805.2369]. 
[24] Q. Liu, N. H. Christ, and C. Jung, Light Quark Mass Reweighting, arXiv: 1206.0080.

[25] M. Lüscher, Local coherence and deflation of the low quark modes in lattice QCD, JHEP 07 (2007) 081, [0706.2298].

[26] R. Babich, J. Brannick, R. Brower, M. Clark, T. Manteuffel, et al., Adaptive multigrid algorithm for the lattice Wilson-Dirac operator, Phys.Rev.Lett. 105 (2010) 201602, [arXiv: 1005.3043 ].

[27] A. Frommer, K. Kahl, S. Krieg, B. Leder, and M. Rottmann, Aggregation-based Multilevel Methods for Lattice QCD, PoS LATTICE2011 (2011) 046, [arXiv: 1202 . 2462].

[28] S. D. Cohen, R. Brower, M. Clark, and J. Osborn, Multigrid Algorithms for Domain-Wall Fermions, PoS LATTICE2011 (2011) 030, [arXiv: 1205 . 2933].

[29] L. Del Debbio, G. M. Manca, and E. Vicari, Critical slowing down of topological modes, Phys.Lett. B594 (2004) 315-323, [hep-lat/ 0403001$].$

[30] M. Lüscher, Topology, the Wilson flow and the HMC algorithm, PoS LATTICE2010 (2010) 015, [arXiv:1009.5877].

[31] ALPHA Collaboration, S. Schaefer, R. Sommer, and F. Virotta, Critical slowing down and error analysis in lattice QCD simulations, Nucl.Phys. B845 (2011) 93-119, [arXiv: 1009 . 5228].

[32] A. Deuzeman and U. Wenger, Gradient flow and scale setting for twisted mass fermions, PoS Lattice 2012 (2012) 162.

[33] A. Chowdhury, A. K. De, S. De Sarkar, A. Harindranath, J. Maiti, et al., Exploring autocorrelations in two-flavour Wilson Lattice QCD using DD-HMC algorithm, arXiv:1209.3915.

[34] B. Alles, G. Boyd, M. D’Elia, A. Di Giacomo, and E. Vicari, Hybrid Monte Carlo and topological modes of full QCD, Phys.Lett. B389 (1996) 107-111, [hep-lat/9607049].

[35] SESAM Collaboration, T(X)L Collaboration Collaboration, G. S. Bali et al., Quark mass effects on the topological susceptibility in QCD, Phys.Rev. D64 (2001) 054502, [hep-lat / 0102002 ].

[36] C. Bernard, T. A. DeGrand, A. Hasenfratz, C. E. Detar, J. Osborn, et al., Topological susceptibility with the improved Asqtad action, Phys.Rev. D68 (2003) 114501, [hep-lat/ 0308019 ].

[37] P. Fritzsch, F. Knechtli, B. Leder, M. Marinkovic, S. Schaefer, et al., The strange quark mass and Lambda parameter of two flavor QCD, Nucl.Phys. B865 (2012) 397-429, [arXiv: 1205.5380 ].

[38] H. Fukaya, Lattice QCD with fixed topology, hep-lat/0603008.

[39] JLQCD Collaboration Collaboration, S. Aoki et al., Two-flavor QCD simulation with exact chiral symmetry, Phys.Rev. D78 (2008) 014508, [arXiv: 0803 .3197].

[40] R. Brower, S. Chandrasekharan, J. W. Negele, and U. J. Wiese, QCD at fixed topology, Phys. Lett. B560 (2003) 64-74, [hep-lat/ 0302005$].$

[41] A. Kennedy and B. Pendleton, Cost of the generalized hybrid Monte Carlo algorithm for free field theory, Nucl.Phys. B607 (2001) 456-510, [hep-lat/ 0008020 ].

[42] M. Lüscher and S. Schaefer, Non-renormalizability of the HMC algorithm, JHEP 1104 (2011) 104, [arXiv:1103.1810].

[43] J. Zinn-Justin, Renormalization and stochastic quantization, Nucl.Phys. B275 (1986) 135.

[44] L. Baulieu and D. Zwanziger, QCD(4) from a five-dimensional point of view, Nucl.Phys. B581 (2000) 604-640, [hep-th/9909006]. 
[45] G. P. Engel and S. Schaefer, Testing trivializing maps in the Hybrid Monte Carlo algorithm, Comput.Phys.Commun. 182 (2011) 2107-2114, [arXiv: 1102.1852 ].

[46] M. Lüscher and S. Schaefer, Lattice QCD without topology barriers, JHEP 1107 (2011) 036, [arXiv:1105.4749].

[47] R. Narayanan and H. Neuberger, Infinite N phase transitions in continuum Wilson loop operators, JHEP 0603 (2006) 064, [hep-th / 0601210 ].

[48] M. Lüscher, Properties and uses of the Wilson flow in lattice QCD, JHEP 1008 (2010) 071, [arXiv:1006.4518].

[49] M. Lüscher and P. Weisz, Perturbative analysis of the gradient flow in non-abelian gauge theories, JHEP 1102 (2011) 051, [arXiv:1101.0963].

[50] S. Borsanyi, S. Durr, Z. Fodor, C. Hoelbling, S. D. Katz, et al., High-precision scale setting in lattice QCD, JHEP 1209 (2012) 010, [arXiv:1203.4469].

[51] A. M. Horowitz, A Generalized guided Monte Carlo algorithm, Phys.Lett. B268 (1991) 247-252. 